\title{
ON THE TRANSLATES OF A SET WHICH MEET IT IN A SET OF POSITIVE MEASURE
}

\author{
JAMES FORAN
}

(Communicated by Andrew Bruckner)

\begin{abstract}
Given a singular Borel regular measure $m_{a}$ on $R^{n}$ and a Borel subset $E$ of $R^{n}$, it is shown that the set of vectors $x$ for which $m_{a}((E+x) \cap E)>0$ is of Lebesgue measure 0 . This fact is then used to show that subsets of finite, nonzero, Hausdorff $s$-measure are nonmeasurable sets with respect to any approximating measure $s-m_{\delta}$.
\end{abstract}

Given a set $E \subset R^{n}$, let $E+x$ denote the translation of $E$ by the vector $x$; that is, $E+x=\{y:$ there is $t \in E$ and $y=t+x\}$. If $m_{a}$ is a measure on $R^{n}$, let $T_{E}\left(m_{a}\right)=\left\{x: m_{a}(E \cap(E+x))>0\right\}$. Let $m(A)$ denote the $n$ dimensional Lebesgue measure of $A$. Theorem 1 shows that $m\left(T_{E}\left(m_{a}\right)\right)=0$ when $m_{a}$ is a singular, $\sigma$-finite, Borel regular measure on $R^{n}$ and $E \subset R^{n}$ is a Borel set. A specific application of and motivation for the result is Theorem 2, which shows that, for $s<n$ and $\delta>0$, every $s$-measurable set with nonzero, finite measure (such sets are called $s$-sets) is a nonmeasurable set with respect to $s-m_{\delta}$. Here, the outer measures $s-m_{\delta}^{*}$ and $s-m^{*}$ are given by $s-m_{\delta}^{*}(E)=$ $\inf \sum \operatorname{diam}\left(E_{i}\right)^{s}$ where the infimum is over all sequences $\left\{E_{i}\right\}$ with $E \subset \bigcup E_{i}$ and each $\operatorname{diam}\left(E_{i}\right)<\delta ; s-m^{*}(E)=\lim _{\delta \rightarrow 0^{+}} s-m_{\delta}^{*}(E)$. Of course, all Borel sets are $s$-measurable, and, if $s-m(E)=0$, then each $s-m_{\delta}(E)=0$ and $E$ is measurable with respect to $s-m_{\delta}$.

Given a set $E \subset R^{n}, E^{\mathrm{c}}$ will denote the complement of $E$ with respect to $R^{n}$. Also, $C_{E}(x)$ will denote the characteristic function of $E$; that is, $C_{E}(x)=1$ if $x \in E$, and $C_{E}(x)=0$ if $x \in E^{\mathrm{c}}$. The symbol $\int$ will denote the integral over all of $R^{n}$.

Theorem 1. Let $m_{a}$ be a singular, $\sigma$-finite, Borel regular measure on $R^{n}$. Let $A$ be a Borel set with $m(A)=0$ so that $m_{a}\left(A^{\mathrm{c}}\right)=0$. Then $m\left(T_{A}\left(m_{a}\right)\right)=0$, and thus, for any Borel set $E, m\left(T_{E}\left(m_{a}\right)\right)=0$.

Proof. For $x \in R^{n}$, define $f(x)=m_{a}(A \cap(A+x))$ where $A$ is the Borel set with $m(A)=0$ and $m_{a}\left(A^{\mathrm{c}}\right)=0$. Since $A$ is a Borel set, so is $A+x$; thus, $C_{A}(t) \cdot C_{A}(t+x)=g(x, t)$ is a measurable function with respect to the product

Received by the editors July 23, 1992 and, in revised form, October 28, 1992; presented at the Summer Symposium on Real Analysis, Acadia University, Wolfville, Nova Scotia, August 13-15, 1992.

1991 Mathematics Subject Classification. Primary 28A78; Secondary 26A21, 28 A05.

Key words and phrases. Borel regular measure, Hausdorff $s$-dimensional measure. 
measure $m \times m_{a}$. Furthermore, $f(x)=m_{a}(\{t: t \in A$ and $t+x \in A\})=$ $\int C_{A}(t) C_{A}(t+x) d m_{a}(t)$ is a nonnegative measurable function. (Cf. [2, pp. 82 $\mathrm{ff}]$ for this fact and the generalized Fubini's theorem for product spaces used below.) To prove the theorem, it suffices to show that $f(x)=0$ except on a set of Lebesgue measure 0 . Since $f(x) \geq 0$, this can be obtained by showing that $\int f(x) d m(x)=0$. But

$$
\begin{aligned}
\int f(x) d m(x) & =\int\left(\int C_{A}(t) C_{A}(t+x) d m_{a}(t)\right) d m(x) \\
& =\int C_{A}(t)\left(\int C_{A}(t+x) d m(x)\right) d m_{a}(t) \\
& =\int C_{A}(t) \cdot 0 d m_{a}(t)=0 .
\end{aligned}
$$

Note. The above argument is parallel to one frequently used to show that the set of all distances between points of a Lebesgue measurable set of positive measure contains an interval. A slight variation applies to any measure $m_{b}$ which is absolutely continuous with respect to Lebesgue measure. It shows that $T_{E}\left(m_{b}\right)$ must contain an open set when $m_{b}(E)>0$. This is because a compact set $F \subset E$ with $0<m_{b}(F)<\infty$ gives rise to the continuous function

$$
f(x)=\int C_{F}(x+t) C_{F}(t) d m_{b}(t)=\int C_{F}(x+t) C_{F}(t) g(t) d t
$$

where $g$ is the Radon-Nikodym derivative of $m_{b}$ with respect to $m$. But

$$
\begin{aligned}
\int f(x) d x & =\iint C_{F}(x+t) C_{F}(t) g(t) d t d x \\
& =\int C_{F}(t) g(t)\left(\int C_{F}(x+t) d x\right) d t \\
& =m(F) \cdot m_{b}(F)>0 .
\end{aligned}
$$

This is because $m_{b}(F)>0$ and $m_{b}$ absolutely continuous with respect to $m$ implies $m(F)>0$. This argument also implies that $f(x)$ is greater than 0 on an open set $G$, and thus $T_{E}\left(m_{b}\right)$ contains $G$. By the Lebesgue decomposition theorem, this will also hold for any such measure which has a nonzero, absolutely continuous component measure.

Theorem 2. Suppose $E$ is an $s$-set in $R^{n}$ with $s<n$. Then for any $\delta>0, E$ is a nonmeasurable set with respect to the measure $s-m_{\delta}$.

Proof. For each set $X \subset R^{n}$, let $m_{a}^{*}(X)=s-m^{*}(E \cap X)$. Then $m_{a}$ is a singular, Borel regular, $\sigma$-finite measure on $R^{n}$, and, by Theorem $1, m\left(T_{E}\left(m_{a}\right)\right)=0$. Thus the complement of $T_{E}\left(m_{a}\right)$ is dense and contains vectors $x$ of arbitrarily small norm so that $s-m((E+x) \cap E)=0$. Let $\left\{E_{i}\right\}$ satisfy for each $i$ $\operatorname{diam}\left(E_{i}\right)<\delta, E \subset \cup E_{i}$, and $\sum \operatorname{diam}\left(E_{i}\right)^{s}<\frac{5}{4} s-m_{\delta}^{*}(E)$. We may also assume that the $E_{i}$ are open sets. There is $M>0$ so that if $E_{M}=\{x \in E:\|x\| \leq M\}$ then

$$
\sum^{\prime} \operatorname{diam}\left(E_{i}\right)^{s}<\frac{1}{4} s-m_{\delta}^{*}(E)
$$

where $\sum^{\prime}$ is over all $E_{i}$ which contain points $x$ with $\|x\|>M$. Then $s-m_{\delta}^{*}\left(E \backslash E_{M}\right)<\frac{1}{4} s-m_{\delta}^{*}(E)$. By a well-known property of $s$-measure (cf. [1]) 
there is an increasing sequence of compact sets $\left\{F_{n}\right\}$ with $F_{n} \subset E_{M}$ so that $s-m\left(\bigcup F_{n}\right)=s-m\left(E_{M}\right)$. By the increasing sets lemma (cf. [1, pp. 70ff]) there is $N$ so that $F=F_{N}$ satisfies $s-m_{\delta}^{*}(F)>\frac{3}{4} s-m_{\delta}^{*}\left(E_{M}\right)$; thus, since $s-m_{\delta}^{*}(E) \leq$ $s-m_{\delta}^{*}\left(E \backslash E_{M}\right)+s-m_{\delta}^{*}\left(E_{M}\right)$, it follows that

$$
\begin{aligned}
s-m_{\delta}^{*}\left(E_{M}\right) & \geq s-m_{\delta}^{*}(E)-s-m_{\delta}^{*}\left(E \backslash E_{M}\right) \\
& \geq s-m_{\delta}^{*}(E)-\frac{1}{4} s-m_{\delta}^{*}(E) \geq \frac{3}{4} s-m_{\delta}^{*}(E) .
\end{aligned}
$$

Thus $s-m_{\delta}^{*}(F) \geq \frac{3}{4} s-m_{\delta}^{*}\left(E_{M}\right) \geq\left(\frac{3}{4}\right)^{2} s-m_{\delta}^{*}(E)$ and $s-m_{\delta}^{*}(F)>\frac{1}{2} s-m_{\delta}^{*}(E)$. Let $x$ be a vector so that $F+x \subset \bigcup E_{i}$ and $s-m((F+x) \cap F)=0=s-m_{\delta}(F+x \cap F)$. To show that $E$ is not measurable, let $A=E \cup(F+x)$. Since $A \subset \cup E_{i}$ and $s-m_{\delta}^{*}$ is translation invariant,

$$
\begin{aligned}
s-m_{\delta}^{*}(A) & \leq \sum \operatorname{diam}\left(E_{i}\right)^{s} \leq s-m_{\delta}^{*}(E)+\frac{1}{4} s-m_{\delta}^{*}(E) \\
& \leq s-m_{\delta}^{*}(E)+\frac{1}{2} s-m_{\delta}^{*}(F) \\
& \leq s-m_{\delta}^{*}(E)+\frac{1}{2} s-m_{\delta}^{*}(F+x) \\
& <s-m_{\delta}^{*}(A \cap E)+s-m_{\delta}^{*}(A \backslash E) .
\end{aligned}
$$

Thus, by the standard criterion for measurability, $E$ is not measurable.

Note that the above argument could be generalized to measures determined by a nondecreasing continuous function $h$ defined on $(0, \infty)$ with $\lim _{t \rightarrow 0^{+}} h(t)=$ 0 where $h-m_{\delta}^{*}$ is defined by replacing $\operatorname{diam}(E)^{s}$ with $h(\operatorname{diam}(E))$.

While Theorem 1 guarantees that the Lebesgue measure of $T_{E}(s-m)$ is zero whenever $E$ is an $s$-set, several natural questions arise as to whether the size of $T_{E}(s-m)$ is not considerably smaller. In particular,

(1) For $s$-sets $E$ in $R^{n}$ with $n>s$ (especially when $s$ is not a whole number), what is the supremum over all $E$ of $\operatorname{dim}\left(T_{E}(s-m)\right)$ where $\operatorname{dim}(X)=$ $\inf \{s: s-m(X)=0\}$ ?

(2) If $E$ is an $s$-set in $R^{n}$ where $s$ is not a whole number, is it possible that $E+E=\{z: t=x+y, x \in E, y \in E\}$ is also an $s$-set?

\section{REFERENCES}

1. C. A. Rogers, Hausdorff measures, Cambridge Univ. Press, Cambridge and New York, 1970.

2. S. Saks, Theory of the integral, second revised ed., Dover, New York, 1964.

Department of Mathematics, University of Missouri-Kansas City, Kansas City, MisSOURI 64110

E-mail address: jforan@umkcvax1.bitnet 